\title{
COVID-19 pneumonia with back pain: Presentation of an acute pulmonary embolism associated with novel coronavirus infection in an outpatient setting
}

\author{
Kenyani Davis ${ }^{1}$ \\ ${ }^{1}$ Community Health Center of Buffalo Inc
}

May 11, 2020

\begin{abstract}
In March 2020 a pandemic was declared due to a novel coronavirus strain. The virus can create a hypercoagulable state. This case is one of the first to report the development of a pulmonary embolism in an infected patient with no known risk factors in an outpatient setting.
\end{abstract}

\section{Key Clinical Message}

The Chinese Center for Disease Control and Prevention reported 80\% of coronavirus infections were mild cases, hence most cases will be managed in an outpatient setting. COVID-19 is associated with a hypercoagulable state, therefore further research should be done on outpatient use of anticoagulation for VTE/PE prophylaxis in these patients.

\section{Introduction:}

The emergence of COVID-19 caused by the SARS-CoV-2 Virus from Wuhan, China in December 2019 has created a global pandemic and strained our entire healthcare system around the world ${ }^{(1)}$. As the medical community continues to learn more about this highly contagious respiratory disease, the most common cause of hospitalization is severe respiratory distress ${ }^{(2-3)}$. However, according to the Chinese Center for Disease Control and Prevention, $80 \%$ of cases caused by COVID-19 are mild cases ${ }^{(4)}$. The novel virus has been well described in the evolving literature as causing a proinflammatory and hypercoagulable state with increases in C-reactive protein, D-Dimer, Ferritin, Lactate Dehydrogenase and interleukin levels ${ }^{(5-6)}$. This marked elevation in inflammatory markers can lead to elevations in bradykinin through the Kinin-Kallikrein system causing propagation of the inflammatory process, vascular relaxation and vasodilation ${ }^{(7)}$.

SARS-Cov-2 virus has the ability to cause pulmonary and systemic inflammation leading to multi-organ dysfunction (7-8). The most common complication during an exacerbation of COVID-19 was Acute Respiratory Distress Syndrome (ARDS), respiratory failure, sepsis, acute cardiac injury and heart failure ${ }^{(8)}$. Among 107 COVID-19 patients admitted to an Intensive Care Unit in the North-of-France there was an increase in pulmonary embolisms during their stay in the tertiary care unit ${ }^{(9)}$. In this study, the frequency in pulmonary embolisms in COVID-19 patients were twice as higher than patients admitted to the ICU pre-pandemic with similar severity entrance scores. A case report of a patient presenting to the emergency room with an acute segmental pulmonary embolus associated with a coronavirus infection demonstrated that an association between COVID-19 and PE could exist in patients who have no risk factors. To date, there are no case reports that detail patients without risk factors who present in an outpatient setting with pulmonary embolisms after being diagnosed with COVID-19. This case illustrates the clinical features of 
the development of a pulmonary embolism in a patient after diagnosis with COVID-19 within an outpatient setting.

\section{Case Report}

34-year-old male with a history of Graves' disease status post thyroidectomy one year ago on levothyroxine presents to the primary care office via telehealth appointment on April 2, 2020 for a hospital follow up. Patient reports that on March 17, 2020 he spiked a fever of $1020 \mathrm{~F}$ coupled with myalgias, intermittent non-productive cough and headaches. He took Ibuprofen for the fever and pain which started to improve. On March 21, 2020 his fever returned with a new symptom of non-bloody diarrhea without abdominal pain. His symptoms quickly progressed to generalized weakness and extreme fatigue. He had a positive contact with his father who tested positive for COVID-19 and with a client whom he had contact with at his job in a group home who also tested positive. He continued to worsen, so he sought medical attention in the emergency room on March 22,2020.

Upon presentation to the emergency room (ER) his vital signs were: Temperature (Temp) 102.50F, Heart Rate (HR) $121 \mathrm{bpm}$, Blood Pressure (BP) 128/75 mmHg and Pulse Oxygen (SpO2) 90\% on room air. His physical exam was unremarkable for everything except for noticeable "fatigue". He was found to have a unilateral left lower lobe infiltrate on chest x-ray (CXR) in which he was placed on oxygen at $2 \mathrm{~L}$ nasal cannula. His saturations began to slowly improve. His blood work resulted a normal white blood cell count with a low absolute lymphocyte count; the remaining Comprehensive Metabolic Panel (CMP) was unremarkable with a negative influenza swab. Due to the paucity of testing at the time in Western New York he was not tested for COVID-19. When he was able to maintain $94 \%$ oxygen saturations on room air, he was discharged on oral Azithromycin for 5 days. Given his positive contact he was assumed to have COVID-19 despite the unilateral CXR and encouraged to self-isolate for 14 days.

On April $2^{\text {nd }}$ at his appointment, patient stated that his myalgias and fever had improved since completing his antibiotic and he was feeling much better. However, 2 days ago he developed a new symptom of pleuriticlike chest pain that he described as a "burning sensation" every time he took a deep breath. He complained of mild shortness of breath (SOB) but, no fever, chills, sore throat or cough. He admitted to having severe back pain and described not being able to lay on his right side due to the excruciating pain. His right sided pleuritic chest pain began to worsen which was now accompanied by hematuria. His back pain increased and was unrelieved by muscle relaxants that he took on his own, so he was sent back to the ER later that evening for further work up.

While in the ER his vital signs were as follows: Temp 98.90F, HR $100 \mathrm{bpm}$, BP 107/92, SpO2 96\% on room air. His physical exam was unremarkable as was his complete blood count and cardiac enzymes. His Computed Tomography (CT) Chest with IV contrast demonstrated bilateral air space disease with combination multifocal pneumonia and atelectasis as well as a right lower lobe pulmonary embolus. CT Abdomen/Pelvis disclosed a mildly enlarged prostate but otherwise unremarkable imaging. Patient was discharged on a novel oral anticoagulant. Prior to the patient's diagnosis of a PE he had no known risk factors for PE or Venous Thrombosis Embolism (VTE). He was still mobile after he developed symptoms on March 17th, albeit less than his normal activity but not immobilized.

Patient followed up in office on April $6^{\text {th }}$ in which his symptoms of pleuritic chest pain had resolved. His SOB was improving, and he was without hematuria. Pertinent positives on physical exam included HR 93 bpm, Temp 98.10F, SpO2 90\% with fine crackles in the right lower lung field. The remaining parts of the physical exam were normal. He was later sent for antibody testing in which on April $29^{\text {th }}$ he was positive for SARS-CoV-2 IGG antibodies.

\section{Discussion}

Our understanding of COVID-19 is constantly evolving, but several studies have documented the role of inflammatory markers in patients with COVID-19 that make them more hypercoagulable ${ }^{(3,7,8)}$. Studies out of France have demonstrated that patients with COVID-19 are more likely to develop pulmonary emboli 
within 6 days in the ICU compared to patients with similar disease severity who were admitted prior to the pandemic ${ }^{(9)}$. It was also noted that the frequency of pulmonary emboli in COVID-19 patients was higher than patients admitted to the Intensive Care Units (ICU) with Influenza from January to December 2019. Another research study has suggested that patients may have a mortality benefit from anticoagulation $(2,10)$. One study detailed the relationship between human angiotensinogen-converting enzyme (ACE) and COVID-19. The increased ACE/ACE2 ratio that happens in COVID-19 infections potentially influence kidney damage and decreases the hydrolyzing effects of bradykinin within the Kinin-Kallikrein system ${ }^{(7)}$.

This is pertinent to our case given the fact that our patient potentially developed a PE roughly 14 days after the onset of his first symptoms and 9 days after his diagnosis of COVID-19. He also presented to the emergency room with new onset hematuria with a relatively benign CT Pelvis. His presentation of PE and hematuria can potentially be explained by the biochemical imbalance of ACE/ACE2. Unfortunately, inflammatory markers such as D-Dimer, ESR or Ferritin were not completed, but his presentation of hematuria and new onset PE is likely a result of the brewing cytokine storm at the time. It is not uncommon for people with pneumonia to present with back pain or pleuritic like chest pain due to the focal lesion. The unique finding in our patient is that he started to have back pain two days prior to his appointment, but it was on the opposite side of his pneumonia. What complicates our patient is that his new back pain could be related to an evolving COVID-19 pneumonia in which he may have just now began to develop the bilateral nature of the pneumonia. He had no risk factors for VTE/PE and his description of "burning pain" with breathing could be described in patients with COVID-19 as well.

Coronavirus is normally described as causing a bilateral pneumonia; however, our patient did not present with bilateral disease on his first presentation to the ER. Research has shown that CXR is insensitive in the detection of positive COVID-19 cases in the early phase of the disease ${ }^{(11)}$. This means that our patient on March $22^{\text {nd }}$ most likely was in the early phase of the disease process. The study from France, reported the development of a PE amongst critically ill patients within a median time from ICU admission of 6 days, but ranging from 1 to 18 days ${ }^{(9)}$. PE development appears to occur in non-hospitalized patients with moderate COVID-19 symptoms within an outpatient setting as well as demonstrated in our case. Unlike the patients in the ICU, our patient was mobile which further strengthens the previous research studies identifying the hypercoagulable state of these patients.

It would have been ideal if our patient was tested for COVID-19 at his first presentation to the emergency room however the lack of testing capacity within the region precluded him from being tested. Eventually, he dawned the pathognomonic signs of COVID-19 by having a bilateral pneumonia on CT Chest imaging on April $2^{\text {nd }}$. Followed by a positive antibody test roughly 6 weeks after the first onset of symptoms which all together corroborates the original clinical diagnosis of COVID-19 on March $22^{\text {nd }}$.

\section{Conclusion}

This case is one of the first to report the development of a PE in a sequential fashion of a patient infected with SARS-CoV-2 with mild-to-moderate COVID-19 symptoms with no known VTE risk factors in an outpatient setting. Awareness of the potential timeline in which patient's develop PE's is important for outpatient clinicians to be aware of so they can better triage their patients and prevent adverse outcomes. Further research should be done on the usage of anticoagulant medications in an outpatient setting for VTE/PE prophylaxis in mild-to-moderate COVID-19 infections for a short course during the active phase.

\section{Authorship}

Author 1: Kenyani Davis, MD, MPH is a General Internal Medicine/Preventive Medicine Physician at the Community Health Center of Buffalo, Inc. She was directly involved in the care of the patient in the above case report.

\section{Acknowledgements}

Special thank to Arvela Heider, PhD, LaVonne Ansari, PhD, Shilpa Kapoor, DDS and Shahla Ahmad, MD for their editorial contributions. 


\section{Conflict of Interest Statement}

There are no conflicts of interests

\section{Reference:}

1. WHO Timeline-COVID 19. (2020, April27). Retrieved from https://www.who.int/newsroom/detail/27-04-2020-who-timeline - covid-19

2. American journal of emergency medicine PE case report

3. Guan W.J., Ni Z. Y., Liang W.H., Ou C Q. Clinical characteristics of coronavirus disease 2019 in China. N. Engl. J. Med. 28 Feb 2020 [Google Scholar]

4. Wu Z, McGoogan JM. Characteristics of and Important Lessons From the Coronavirus Disease 2019 (COVID-19) Outbreak in China: Summary of a Report of 72314 Cases From the Chinese Center for Disease Control and Prevention. Jama 2020.

5. Chen G., Wu D., Cao Y., Huang D. Clinical and immunologic features in severe and moderate coronavirus disease 2019. J. Clin. Invest. 2020 Mar 27 (pii:137244) [Google Scholar]

6. Han H., Yang L., Liu R., Liu F., Wu K.L. Prominent changes in the blood coagulation of the patients with SARS-CoV-2 infection. Clin. Chem Lab Med (CCLM) Mar 2020 [Google Scholar]

7. Tolouian, R., Vahed, S. Z., Ghiyasvand, S., Tolouian, A., \& Ardalan, M. (2020). COVID-19 interactions with angiotensin-converting enzyme 2 (ACE2) and the kinin system; looking at a potential treatment. Journal of Renal Injury Prevention , 9 (2). doi: 10.34172/jrip.2020.19

8. Chen, T., Wu, D., Chen, H., Yan, W., Yang, D., Chen, G., .. N Ning, Q. (2020). Clinical characteristics of 113 deceased patients with coronavirus disease 2019: retrospective study. Bmj , m1091. doi: 10.1136/bmj.m1091

9. Poissy, J., Goutay, J., Caplan, M., Parmentier, E., Duburcq, T., Lassalle, F., ... Susen, S. (2020). Pulmonary Embolism in COVID-19 Patients: Awareness of an Increased Prevalence.Circulation . doi: 10.1161/circulationaha.120.047430

10. Tang Nig. Anticoagulant treatment is associated with decreased mortality in severe coronavirus disease 2019 patients with coagulopathy. J. Thromb. Haemost. 27 Mar. 2020 [Google Scholar] 